\title{
Phosphoglycerate kinase 1 as a promoter of metastasis in colon cancer
}

\author{
SUFIAN S. AHMAD ${ }^{1 *}$, JÖRG GLATZLE ${ }^{*}$, KHALED BAJAEIFER $^{1}$, SARAH BÜHLER $^{3}$, THORSTEN LEHMANN $^{1}$, \\ INGMAR KÖNIGSRAINER ${ }^{1}$, JÖRG-PETER VOLLMER ${ }^{2}$, BENCE SIPOS ${ }^{2}$, SUHAIB S. AHMAD ${ }^{1}$, \\ HINNAK NORTHOFF $^{3}$, ALFRED KÖNIGSRAINER $^{1}$ and DEREK ZIEKER ${ }^{1}$ \\ ${ }^{1}$ Department of General, Visceral and Transplantation Surgery, ${ }^{2}$ Institute of Pathology and Neuropathology, \\ ${ }^{3}$ Institute for Clinical and Experimental Transfusion Medicine, University of Tübingen, D-72076 Tübingen, Germany
}

Received February 19, 2013; Accepted April 12, 2013

DOI: 10.3892/ijo.2013.1971

\begin{abstract}
Oxidative stress due to intratumoral hypoxia in solid cancer has been shown to be associated with increased mortality. Phosphoglycerate kinase 1 (PGK1) is an enzyme of the glycolytic pathway, which is regulated by hypoxia-inducible factor-1 $\alpha$ (HIF-1 $\alpha$ ) and has been described for its role in tumor progression and metastasis in several malignancies. We investigated whether the expression of PGK1 varies between metastatic and non-metastatic colon cancer. We compared PGK1 expression in colon cancer patients either with or without metastasis via polymerase chain reaction (PCR) and immunohistochemistry. Microarray analysis was performed to test altered gene expression after PGK1 silencing, using isolates from HCT116 cell lines. PCR results showed an increased expression of PGK1 in colon cancer tissue from metastatic patients in comparison to patients with no metastasis (fold change 2.6, $\mathrm{p}<0.001)$. Immunohistochemical staining of PGK1 showed stronger staining in metastatic tissue in comparison to non-metastatic cancer tissue according to a semi-quantitative evaluation. Microarray and subsequent pathway analysis provided 4 genes of interest (CYR61, FOS, JUN and EGR1) used for pathway proposal. The results indicate that increased expression of PGK1 in colon cancer tissue is associated with metastasis. Furthermore, we propose several genes induced by PGK1 that could account for cell migration, mainly EGR1 and CYR61 together with the transcription factors FOS and JUN.
\end{abstract}

Correspondence to: Dr Jörg Glatzle, Department of General, Visceral and Transplantation Surgery, University of Tübingen, Hoppe Seyler Str. 3, D-72076 Tübingen, Germany

E-mail: joerg.glatzle@med.uni-tuebingen.de

*Contributed equally

Key words: phosphoglycerate kinase 1, colon cancer, cancer metastasis, hypoxia, tumor hypoxia, tumor invasion, lymph node metastasis

\section{Introduction}

Colorectal cancer (CRC) presents a problematic issue in the developed world as it is the major cause of cancer-related death. Over 1.2 million new cases were diagnosed in 2008 and 600,000 deaths were reported (1). There has been considerable interest in understanding carcinogenesis of CRC throughout the last decades. Hereditary and sporadic forms of colon cancer have been reported (2). Germline mutations in the APC gene and the DNA mismatch repair complex are described as the genetic explanations for inherited familial adenomatous polyposis syndrome and hereditary non-polyposis colon cancer (HNPCC) respectively (3-6). Sporadic cancers of the colon are associated with K-ras, TGF- $\beta$ receptor, p53 and somatic APC mutations $(3,6-8)$.

Oxidative stress has been implicated in a number of colon pathologies including inflammatory bowel disease, diverticulitis and cancer (9-11). Tumor progression is greatly regulated by hypoxia, the bulky nature of a tumor distances the cells from oxygen-rich blood vessels, which implicates cellular response (12). Intratumoral hypoxia has been associated with increased risk of mortality (13). Oxidative stress allows for the activation of several gene products that would promote metabolic adaptation to hypoxia, such as those responsible for angiogenesis and glycolytic enzymes (14-16). Hypoxia inducible factors (HIFs) function physiologically in adaptation to hypoxic conditions, they present the most notable group assciated with hypoxiainduced reactions (17).

The phosphoglycerate kinase 1 (PGK1) is an ATPgenerating enzyme of the glycolytic pathway catalyzing the conversion of 1,3-diphosphoglycerate to 3-phosphoglycerate and regulated by hypoxia-induced factor- $1 \alpha$ (HIF-1 $\alpha$ ), which is the most important factor involved in the cellular response to hypoxia $(18,19)$. Solid tumor cells employ glycolytic enzymes such as PGK1, to generate ATP when their supply of oxygen is limited (20). Several malignancies including prostate cancer, breast cancer, pancreatic ductal adenocarcinoma, multidrugresistant ovarian cancer and as we have demonstrated, metastatic gastric cancer, have all been shown to exhibit an increased expression of PGK1 (21-25). An increased interest in PGK1 has arisen due to its possible role in invasion and metastasis; several possible pathways have been described, 
including the chemokine axis (26), allowing for an indirect impact of PGK1 on migration, angiogenesis and tumor growth (27). Furthermore, $\beta$-catenin, a molecule involved in cell proliferation, invasion, metastasis, angiogenesis and drug resistance, seems to be a downstream target of PGK1 $(18,28,29)$. The expression of PGK1 has not yet been investigated in metastatic colon cancer. In this study, we examined whether the PGK1 expression varies between metastatic and non-metastatic colon cancer to find potential pathways accounting for such observations.

\section{Patients and methods}

Patients, tissue specimens and RNA extraction. Tissue samples were obtained from patients who underwent hemi- or total colectomy at the Department of General, Viszeral- and Transplant Surgery of the University of Tübingen, Germany. Manual micro-dissection and harvesting was performed immediately after tumor resection to ensure high tumor cell content of the samples, specimens were then snap-frozen in liquid nitrogen and stored at $-80^{\circ} \mathrm{C}$ until utilization. Only specimens from patients with histologically confirmed diagnosis of adenocarcinoma were considered. A total of 27 samples (17 males and 10 females) were utilized for RNA extraction. Thirteen from patients without metastasis (mean age, 68 years; range, 52-80; pT1-category, 2; pT2-category, 2; pT3-category, 9) and 14 from patients with histologically confirmed metastasis of at least N1 category according to UICC (30) (mean age, 69 years; range, 51-92; pT2-category, 1; pT3-category, 9; pT4-category, 4; N1, 4; pN1-category, 4; pN2-category, 6; pN3-category, 4; M0-category, 8; M1-category, 6). All patients signed an informed written consent to participate in the study.

RNA extraction was performed using the RNAeasy ${ }^{\circledR}$ Mini kit (Qiagen, Hilden, Germany) according to the manufacturer's instructions. The quality and quantity of the mRNA yield was monitored using the NanoDrop ND-1000 spectrophotometer (NanoDrop Technologies, Wilmington, DE, USA).

Specimens used for immunhistochemical staining were retrieved from a tissue bank of the comprehensive cancer center of the University of Tübingen, Germany. Twenty-seven paraffin-embedded tissue blocks of adenocarcinoma samples were randomly chosen with the help of a corresponding database, 17 from non-metastatic colon cancer patients (pT2-category, 3; pT3-category, 14; localization: 9 ascending colon, 2 transverse colon, 6 descending colon) and 10 from metastatic colon cancer patients (pT2-category, 2; pT3-category, 8; pN1-category, 7; pN2-category, 3; M1-category, 2; tumor localization: 5 ascending colon, 2 transverse colon, 2 descending colon, 1 recto-sigmoid region).

Real-time LightCycler ${ }^{\circledR} R T$-PCR. cDNA was prepared using the first-strand cDNA synthesis kit (Roche Applied Science, Mannheim, Germany) according to the manufacturer's instructions. Real-time PCR was then performed with the SYBR Green Jump Start TAQ ReadyMix (Sigma, Taufkirchen, Germany) and LightCycler (Roche Applied Science) as described previously (26). The number of specific transcripts was normalized to $\beta$-actin as a housekeeping gene. For analysis of relative expression, the $2^{-\Delta \Delta \mathrm{T}}$ method was utilized according to Livak and Schmittinger (31). The Mann-Whitney
$\mathrm{U}$ test was used for comparison of expression data, $\mathrm{p}<0.05$ was considered statistically significant. Sequences of the primers used in this study are listed in Table I.

Immunohistochemistry. For immunhistochemistry of colon cancer samples, serial paraffin sections $(2 \mu \mathrm{m})$ of the selected tumor blocks were cut and deparaffinized. After protease antigen retrieval (6 min), PGK1 staining with PGK1/2 primary antibody (dilution 1:200; clone: sc-48342; Santa Cruz Biotechnology Inc., Santa Cruz, CA, USA) was carried out on an automated immunostainer (Benchmark, Ventana Medical Systems, USA) with an incubation time of $32 \mathrm{~min}$. Counterstaining was performed using a biotin-free ultraView Universal DAB Detection kit (Ventana Medical Systems). Semi-quantative analysis was performed by 2 pathologists based on the intensity of tumor cell staining.

Cell culture. The HCT116 (ATCC Manassas, VA, USA) cell line was utilized for PGK1 knockdown and microarray investigations as described below. Cells were cultured in Dulbecco's modified Eagle's medium (DMEM) (Lonza, Basel, Switzerland) $+10 \%$ fetal calf serum (FCS) (PAA, Pasching, Austria) and maintained in a humid chamber at a $37^{\circ} \mathrm{C}$ and $5 \% \mathrm{CO}_{2}$ atmospheric condition.

Small hairpin RNA knockdown of PGK1. HCT cells were incubated with an adenovirus (Sirion Biotech, Martinsried, Germany) containing shPGK1 for $48 \mathrm{~h}$. RNA was then isolated using the RNAeasy Mini kit (Qiagen) and quantified with the NanoDrop ND-1000 spectrophotometer (NanoDrop Technologies).

Microarray analysis. To generate gene expression profiles, both steady state and actively translated RNA was isolated from wild-type HCT116 and PGK1-silenced HCT116 cell lines. Three aliquots containing $10 \mu \mathrm{g}$ RNA each were harvested from both cell lines and quantified using the NanoDrop ND-1000 spectrophotometer (NanoDrop Technologies). The GeneChip 3' IVT Express kit was used to synthesize biotinylated amplified RNA (aRNA) that was hybridized to the Affymetrix GeneChip ${ }^{\circledR}$ human genome U133 Plus 2.0 array (Affymetrix Inc., Santa Clara, CA, USA). In brief, first strand cDNA was synthesized via reverse transcription, then converted into a double stranded template for transcription. In vitro transcription allowed for synthesis of aRNA incorporating a biotin-conjugated nucleotide. aRNA was then fragmented and hybridized onto the Chip Arrays according to the manufacturer's instructions. Chips were washed and stained using a Fluidics Station and scanned with a GeneArray Scanner 3000, both provided by the GeneChip ${ }^{\circledR}$ System 3000Dx v. 2 by Affymetrix. Scanned images were inspected visually to check for hybridization artifacts and proper grid alignment. Images were quantifed to produce transcript level data using the Affymetrix Microarray Analysis Suite (MAS) 5.0, which were then loaded to GeneSpring Software 7.2 (Agilent, Palo Alto, CA, USA) and normalized across arrays. Principal component analysis was performed to test dissociation between the groups. Fold change was derived as the ratio of average differences from the three experimental arrays as compared to the three control arrays. Multiple testing correction was performed and 
Table I. Genes and corresponding primers used for real-time PCR.

Gene product $\quad$ Sense primer $\quad$ Antisense primer

\begin{tabular}{lll}
\hline Phosphoglycerate kinase 1 (PGK1) & CATACCTGCTGGCTGGATGG & CCCACAGGACCATTCCACAC \\
Early growth response 1 (EGR1) & CTGACCGCAGAGTCTTTTC & AAGGTGTTGCCACTGTTG \\
Jun oncogene (JUN) & TGACGGACTGTTCTATGACT & AAGGTGTTGCCACTGTTG \\
FBJ murine osteosarcoma viral & GCAAGGTGGAACAGTTATCT & TTCAGCAGGTTGGCAATC
\end{tabular}
oncogene homolog (FOS)

Cysteine-rich, angiogenic inducer, 61 GAAGAGTGTCAGAATCAGAATCA TACCTTAATGCTCCTCAAGAATG

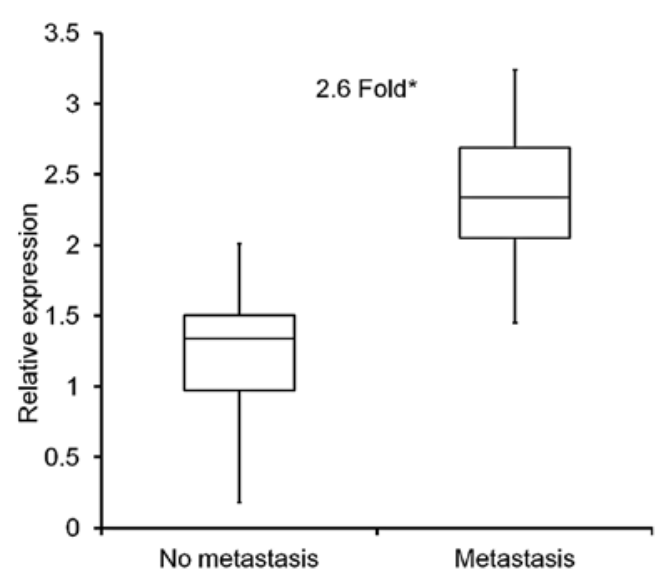

Figure 1. Box-plot of relative PGK1 expression of the metastatic group compared to the non-metastatic group. Fold change (FC), 2.6; ${ }^{*} \mathrm{p}<0.001$.

a 2-fold cutoff was set. Expression profiles were compared using one-way analyses of variance (ANOVA), $\mathrm{p}<0.05$ was considered statistically significant. Web-based Ingenuity Pathway software (Ingenuity ${ }^{\circledR}$ Systems, www.ingenuity.com) was employed to examine the function and pathway of the different genes.

\section{Results}

mRNA expression levels of PGK1 by means of QRT-PCR. Quantative real-time PCR (qRT-PCR) was used to assess mRNA expression of PGK1 in colon cancer tissue extracts, either from patients without metastasis $(n=13)$ or with histologically confirmed metastasis $(n=14)$ as mentioned above. PGK1 expression levels were significantly higher in metastatic samples by 2.6 -fold ( $\mathrm{p}<0.001$ ), when compared to non-metastatic colon cancer samples (Fig. 1).

Semi-quantative analysis by means of immunohistochemistry. PGK1 stained sections of 27 colon tumor specimens, either without metastasis $(n=17)$, or with metastasis $(n=10)$ were evaluated by two pathologists. Semi-quantative analysis was performed by assessing the stained tumor area ( $>50 \%$, strong; $<50 \%$, moderate; $<10 \%$, weak). Sections of the metastasis group (9/10) showed strong to moderate staining (6 strong, 3 moderate), 1 section was negative. Whereas $13 / 17$ of the non-metastatic sections showed weak to moderate staining
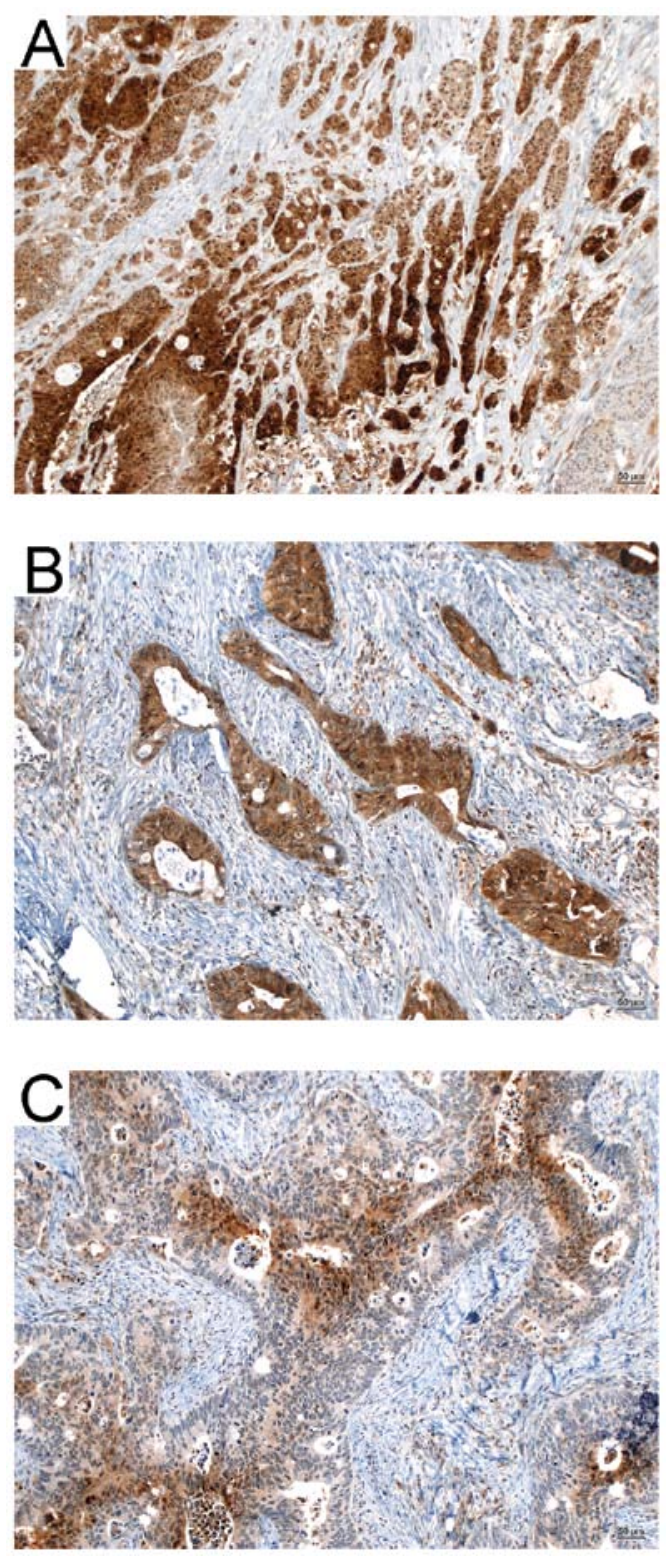

Figure 2. Microscopic images of strong staining in a metastatic patient (A), moderate staining in a metastatic patient (B) and weak staining in a nonmetastatic patient with colon cancer (C).

(1 negative, 6 weak, 6 moderate) and only 4/17 strong. Fig. 2 shows representative histological images. 


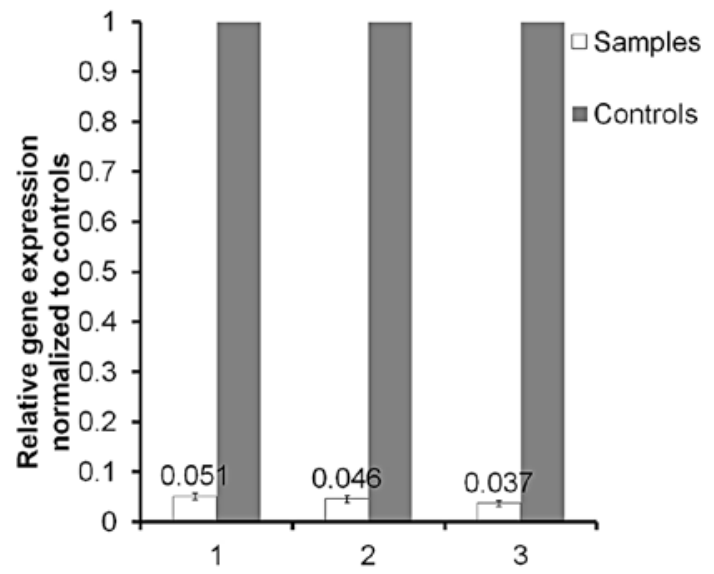

Figure 3. Real-time PCR confirmation of successful silencing of PGK1 in HCT116 cells in comparison to the corresponding wild-type control.

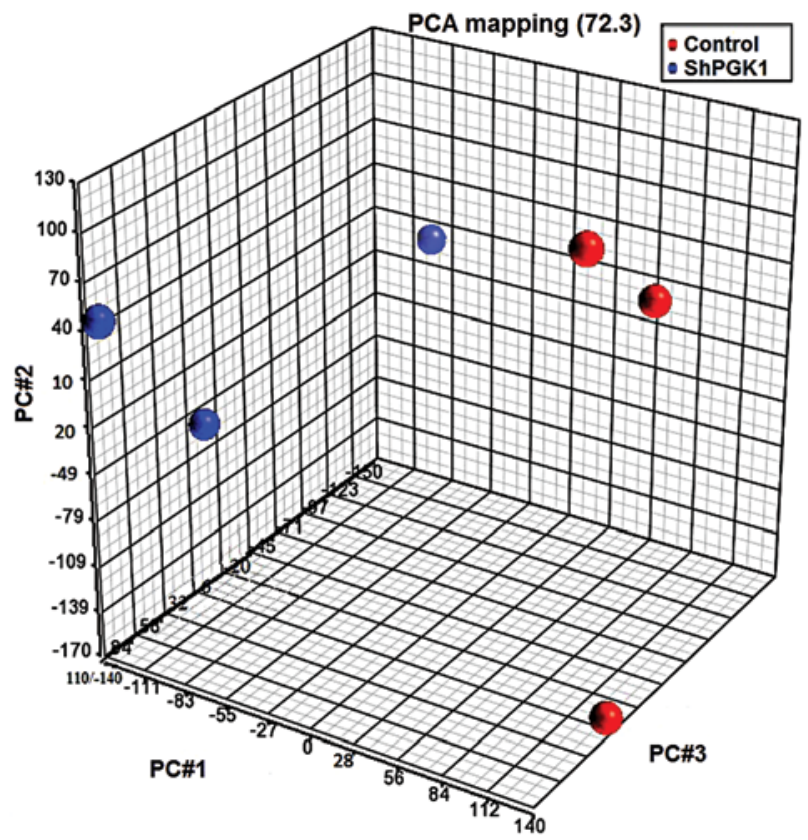

Figure 4. Differential expression levels of transcripts in PGK1 silenced HCT116 (ShPGK1) versus wild-type control HCT116 (control). Each dot in the $3 \mathrm{D}$ visualization represents a sample, not a gene. Principal component analysis (PCA) captured $72.3 \%$ of the variation observed in the experiment in the first three principal components (PC), which are plotted on $\mathrm{x}-, \mathrm{y}-$ and $\mathrm{z}$-axes, respectively, representing the largest fraction of the overall variability in samples.

Microarray analysis and validation. Successful PGK1 knockdown was achieved in HCT116 cells (Fig. 3), from which 3 RNA extracts from 3 individual experiments were utilized for microarray analysis. Comparison was made to corresponding native HCT116 RNA extracts of the same passage. Fig. 4 shows a 3D visualization of the relationships between the samples using PCA, which is based on the expression levels of the probe sets: PGK1 knockdown and native groups clustered in separate areas of the 3-dimentional visualization, indicating a clear difference in the molecular composition between them. PCA captured $72.3 \%$ of the variation observed in the experiment in the first three principal components, which are plotted

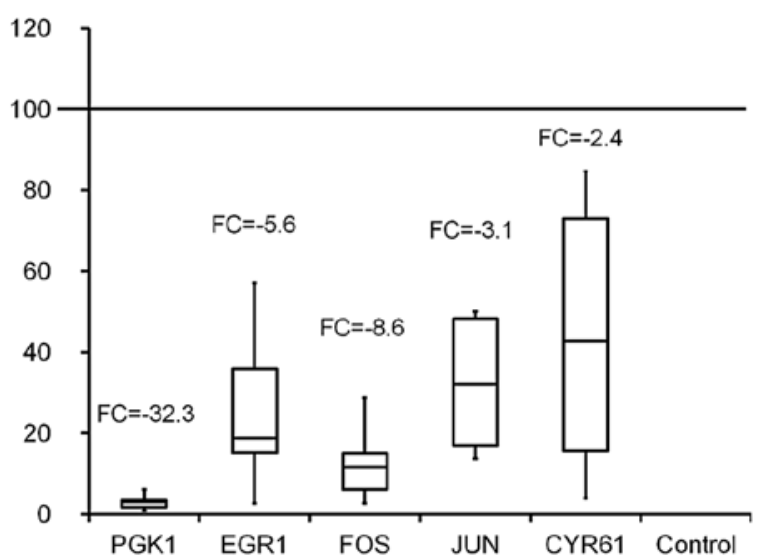

Figure 5. Real-time PCR validation of microarray results showing downregulation of the genes of interest: PGK1, EGR1, FOS, JUN, CYR61 when compared to wild-type control (straight line at 100).

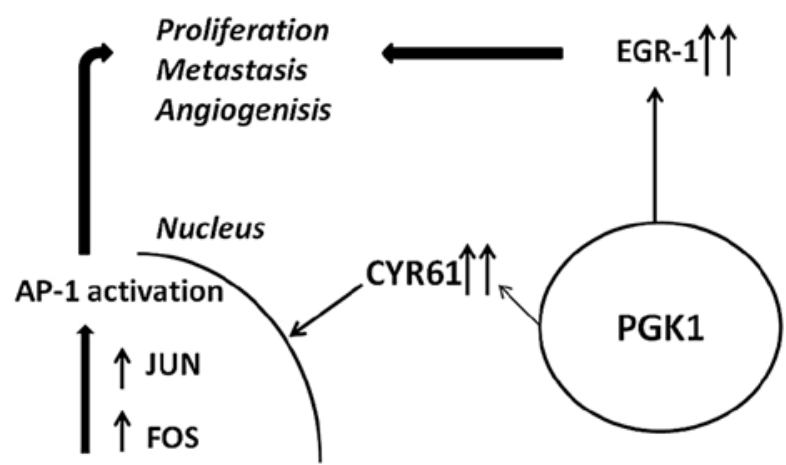

Figure 6. Model of PGK1 signaling. Overexpression of PGK1 signals through increased expression of EGR-1, CYR61 and consequently the transcription factors FOS and JUN, invasion, proliferation and angiogenesis.

on $\mathrm{x}-, \mathrm{y}-$ and $\mathrm{z}$-axes, respectively, representing the largest fraction of the overall variability in samples. At a significance level of $<0.05$ and a cutoff fold change of $\geq 2,92$ genes were upregulated and 180 downregulated. After uploading data sets containing gene identifiers into Ingenuity Pathway software (Ingenuity Systems, www.ingenuity.com), genes of interest were further tested by means of RT-PCR for microarray validation showing downregulation of PGK1 fold change (FC) -32.3; early growth response 1 (EGR1) F -5.6; cysteine-rich 61 (CYR61) FC -2.4; FBJ murine osteosarcoma viral oncogene homolog (FOS) FC -8.6; Jun oncogene (JUN) FC -3.2 (Fig. 5).

\section{Discussion}

The data presented in this study indicate that colon cancer cells that tend to metastasize possess an increased expression of PGK1. This has been shown both by PCR on RNA level and immunhistochemical staining. Previous studies described an increased expression of PGK1 in prostate cancer, breast cancer, pancreatic ductal adenocarcinoma, multidrug-resistant ovarian cancer and as we have previously demonstrated, metastatic gastric cancer (21-25). PGK1 is known to be a downstream target of HIF-1 $\alpha$ which is the major factor regulating cellular response to hypoxia (19). 
The bulky nature of tumor masses and thus the impaired conditions for oxygen diffusion is believed to trigger the induction of PGK1, this allows for ATP production and supplementation of cells under hypoxic conditions (24). Furthermore, PGK1 has been shown to be associated with the induction of several pathways, one of which has been described by Wang and his group demonstrating the relationship between PGK1 and the CXCR4/CXCL12 axis in prostate cancer cell lines (18). These findings were reproduced by our group in metastatic gastric cancer (26) as described earlier, but not colon cancer. Knockdown of PGK1 in HCT116 cancer cells was not seen to cause alteration in CXCR4 or CXCL12 expression according to our microarray results.

Several genes, however, appeared to be linked to PGK1 based on the current data. FOS and JUN form a heterodimeric complex which regulates gene transcription by binding to transcriptional control elements containing activator protein1 (AP-1) binding sites (32). This interaction is known to be induced by CYR61 resulting in invasive growth, migration and angiogenesis (Fig. 6) (33,34).

EGR1, a member of the early growth family has also shown an association with PGK1. Interestingly, it has been indicated that EGR1 plays a role in angiogenesis, growth and metastasis (35). These findings were reproduced by Zheng and his group demonstrating that the majority of gastric cancer tissues expressed EGR1 and that the increased expression was associated with metastasis and growth (36).

In conclusion, the data of this study indicate that an increased expression of PGK1 in colon cancer tissue is associated with metastasis. Furthermore, we propose several genes induced by PGK1 that could account for cell migration, mainly EGR1 and CYR61 together with its downstream transcription factors FOS and JUN $(33,36)$.

\section{References}

1. Jemal A, Bray F, Center MM, Ferlay J, Ward E and Forman D: Global cancer statistics. CA Cancer J Clin 61: 69-90, 2011.

2. Markowitz SD, Dawson DM, Willis J and Willson JK: Focus on colon cancer. Cancer Cell 1: 233-236, 2002.

3. Goss $\mathrm{KH}$ and Groden J: Biology of the adenomatous polyposis coli tumor suppressor. J Clin Oncol 18: 1967-1979, 2000.

4. Kinzler KW and Vogelstein B: Lessons from hereditary colorectal cancer. Cell 87: 159-170, 1996.

5. Kolodner R: Biochemistry and genetics of eukaryotic mismatch repair. Genes Dev 10: 1433-1442, 1996.

6. Markowitz S: TGF-beta receptors and DNA repair genes, coupled targets in a pathway of human colon carcinogenesis. Biochim Biophys Acta 1470: M13-M20, 2000.

7. Fearon ER: K-ras gene mutation as a pathogenetic and diagnostic marker in human cancer. J Natl Cancer Inst 85: 1978-1980, 1993.

8. Markowitz S, Wang J, Myeroff L, et al: Inactivation of the type II TGF-beta receptor in colon cancer cells with microsatellite instability. Science 268: 1336-1338, 1995.

9. Borek C: Dietary antioxidants and human cancer. Integr Cancer Ther 3: 333-341, 2004.

10. Seril DN, Liao J, Yang GY and Yang CS: Oxidative stress and ulcerative colitis-associated carcinogenesis: studies in humans and animal models. Carcinogenesis 24: 353-362, 2003.

11. Rhodes JM and Campbell BJ: Inflammation and colorectal cancer: IBD-associated and sporadic cancer compared. Trends Mol Med 8: 10-16, 2002.

12. Mazure NM, Brahimi-Horn MC, Berta MA, et al: HIF-1: master and commander of the hypoxic world. A pharmacological approach to its regulation by siRNAs. Biochem Pharmacol 68: 971-980, 2004.
13. Semenza GL: Hypoxia-inducible factors: mediators of cancer progression and targets for cancer therapy. Trends Pharmacol Sci 33: 207-214, 2012.

14. Carmeliet P, Dor Y, Herbert JM, et al: Role of HIF-1alpha in hypoxia-mediated apoptosis, cell proliferation and tumour angiogenesis. Nature 394: 485-490, 1998.

15. Ryan HE, Lo J and Johnson RS: HIF-1 alpha is required for solid tumor formation and embryonic vascularization. EMBO J 17: 3005-3015, 1998

16. Seagroves TN, Ryan HE, Lu H, et al: Transcription factor HIF-1 is a necessary mediator of the pasteur effect in mammalian cells. Mol Cell Biol 21: 3436-3444, 2001.

17. Semenza GL: Oxygen sensing, homeostasis and disease. N Engl J Med 365: 537-547, 2011.

18. Wang J, Wang J, Dai J, et al: A glycolytic mechanism regulating an angiogenic switch in prostate cancer. Cancer Res 67: 149-159, 2007.

19. Dayan F, Roux D, Brahimi-Horn MC, Pouyssegur J and Mazure NM: The oxygen sensor factor-inhibiting hypoxiainducible factor-1 controls expression of distinct genes through the bifunctional transcriptional character of hypoxia-inducible factor-1alpha. Cancer Res 66: 3688-3698, 2006.

20. Daly EB, Wind T, Jiang XM, Sun L and Hogg PJ: Secretion of phosphoglycerate kinase from tumour cells is controlled by oxygen-sensing hydroxylases. Biochim Biophys Acta 1691: 17-22, 2004.

21. Migita T, Oda Y, Naito S, Morikawa W, Kuwano M and Tsuneyoshi M: The accumulation of angiostatin-like fragments in human prostate carcinoma. Clin Cancer Res 7: 2750-2756, 2001.

22. Duan Z, Lamendola DE, Yusuf RZ, Penson RT, Preffer FI and Seiden MV: Overexpression of human phosphoglycerate kinase 1 (PGK1) induces a multidrug resistance phenotype. Anticancer Res 22: 1933-1941, 2002.

23. Hwang TL, Liang Y, Chien KY and Yu JS: Overexpression and elevated serum levels of phosphoglycerate kinase 1 in pancreatic ductal adenocarcinoma. Proteomics 6: 2259-2272, 2006.

24. Zhang D, Tai LK, Wong LL, Chiu LL, Sethi SK and Koay ES: Proteomic study reveals that proteins involved in metabolic and detoxification pathways are highly expressed in HER-2/neupositive breast cancer. Mol Cell Proteomics 4: 1686-1696, 2005.

25. Zieker D, Konigsrainer I, Tritschler I, et al: Phosphoglycerate kinase 1 a promoting enzyme for peritoneal dissemination in gastric cancer. Int J Cancer 126: 1513-1520, 2010.

26. Zieker D, Konigsrainer I, Traub F, et al: PGK1 a potential marker for peritoneal dissemination in gastric cancer. Cell Physiol Biochem 21: 429-436, 2008.

27. Gerard $\mathrm{C}$ and Rollins BJ: Chemokines and disease. Nat Immunol 2: 108-115, 2001.

28. Lowy AM, Clements WM, Bishop J, et al: beta-catenin/Wnt signaling regulates expression of the membrane type 3 matrix metalloproteinase in gastric cancer. Cancer Res 66: 4734-4741, 2006.

29. Yamada T, Takaoka AS, Naishiro Y, et al: Transactivation of the multidrug resistance 1 gene by T-cell factor 4/beta-catenin complex in early colorectal carcinogenesis. Cancer Res 60: 4761-4766, 2000.

30. Sobin LH: TNM, sixth edition: new developments in general concepts and rules. Semin Surg Oncol 21: 19-22, 2003.

31. Livak KJ and Schmittgen TD: Analysis of relative gene expression data using real-time quantitative PCR and the 2(-Delta Delta C(T)) method. Methods 25: 402-408, 2001.

32. Curran T and Franza BR Jr: Fos and Jun: the AP-1 connection. Cell 55: 395-397, 1988.

33. Tan TW, Yang WH, Lin YT, et al: Cyr61 increases migration and MMP-13 expression via alphavbeta3 integrin, FAK, ERK and AP-1-dependent pathway in human chondrosarcoma cells. Carcinogenesis 30: 258-268, 2009.

34. Eferl R and Wagner EF: AP-1: a double-edged sword in tumorigenesis. Nat Rev Cancer 3: 859-868, 2003.

35. de Mestre AM, Rao S, Hornby JR, Soe-Htwe T, Khachigian LM and Hulett MD: Early growth response gene 1 (EGR1) regulates heparanase gene transcription in tumor cells. J Biol Chem 280: 35136-35147, 2005.

36. Zheng L, Pu J, Jiang G, et al: Abnormal expression of early growth response 1 in gastric cancer: association with tumor invasion, metastasis and heparanase transcription. Pathol Int 60: 268-277, 2010. 